\title{
COVID-19 Presented with Deep Vein Thrombosis in a Patient with Paroxysmal Nocturnal Haemoglobinuria
}

\author{
Zlatko Pravdic ${ }^{1}$ Mirjana Mitrovic ${ }^{1,2}$ Andrija Bogdanovic ${ }^{1,2}$ Marijana Virijevic ${ }^{1,2}$ Nikica Sabljic ${ }^{1}$ \\ Nikola Pantic ${ }^{1}$ Nada Suvajdzic Vukovic ${ }^{1,2}$ \\ ${ }^{1}$ Clinic of Haematology, University Clinical Centre of Serbia, Belgrade,
Serbia \\ 2 Faculty of Medicine, University of Belgrade, Belgrade, Serbia \\ Address for correspondence Zlatko Pravdic, MD, Clinic of \\ Haematology, University Clinical Centre of Serbia, Koste Todorovica 2, \\ 11000 Belgrade, Serbia (e-mail: zlatko.pravdic@gmail.com).
}

Hamostaseologie 2021;41:397-399.
Abstract
Keywords
- COVID-19
- thrombosis
- PNH
- eculizumab
- anticoagulation

Paroxysmal nocturnal haemoglobinuria (PNH) is a rare, acquired clonal haematological disease characterized by complement-mediated haemolysis, bone marrow failure and venous thrombosis. Anticomplement therapy eculizumab improves survival and reduces complications. Severe acute respiratory distress syndrome corona virus 2 (SARS-CoV-2) disease 2019 (COVID-19) is associated with high incidence of both venous and arterial thrombosis in hospitalized patients with pneumonia. Deep venous thrombosis (DVT) as the presenting symptom of COVID-19 is a rare event. We describe a well-controlled PNH patient on eculizumab for more than 5 years who presented with DVT, while on warfarin, as the first sign of COVID-19. To our knowledge, this is the first described case of DVT in a PNH patient with COVID-19.

\section{Introduction}

Paroxysmal nocturnal haemoglobinuria $(\mathrm{PNH})$ is a rare, acquired clonal haematological disease caused by a somatic mutation of the pig-A gene (Xp22.1) in a clone of a bone marrow stem cell. The result is a defective synthesis of glycosylphosphatidylinositol (GPI)-anchored complement regulatory proteins expressed on erythrocytes. Consequently, PNH erythrocytes are highly susceptible to complementmediated intravascular haemolysis. In addition to haemolysis, PNH is characterized by bone marrow failure and venous thrombosis; arterial thrombosis is far less frequent. $^{1,2}$ Thrombosis is the leading cause of death in $\mathrm{PNH}$ patients. Major risk factors for thrombosis in PNH seem to be PNH clone size and degree of intravascular haemolysis. ${ }^{3}$ However, the basis for thrombosis in PNH is poorly understood. The development of eculizumab, a humanized monoclonal antibody directed against the terminal complement protein $\mathrm{C} 5$, resulted in a dramatic improvement of survival and a reduction in complications.

received

April 7, 2021

accepted after revision

July 19, 2021

Optimal treatment and management of infection by severe acute respiratory distress syndrome corona virus 2 (SARS-CoV-2) disease 2019 (COVID-19) in patients affected by $\mathrm{PNH}$ may be challenging, given the rapid spread of the pandemic and limited literature so far. Specifically, COVID19 is associated with relatively high incidence of both venous and arterial thrombotic events in patients hospitalized for pneumonia. ${ }^{4}$ Median prevalence of venous thromboembolism (VTE) in those patients is approximately $30 \%{ }^{5}$ with deep venous thrombosis (DVT) prevalence of nearly 20\% even on prophylactic doses of anticoagulation. ${ }^{5,6}$ However, DVT as the first sign of COVID-19 is a rare event. ${ }^{7-11}$

We herein describe a case of a well-controlled $\mathrm{PNH}$ patient on eculizumab and warfarin for more than 5 years, who presented with DVT as the first sign of COVID-19.

\section{Case Description}

Our patient is a non-smoker with no comorbidities. In the first quarter of 2009, he had experienced his first episode of

(c) 2021. Thieme. All rights reserved. Georg Thieme Verlag KG,

Rüdigerstraße 14,

70469 Stuttgart, Germany
DOI https://doi.org/

10.1055/a-1554-6432. ISSN 0720-9355. 
Coombs-negative haemolytic anaemia with thrombosis of superior sagittal sinus, treated with anticoagulants for 2 years. At the end of 2013, six months before the presentation that led to diagnosis, he had noticed reddish urine. Midway through 2014, he presented with haemolysis and splenic vein thrombosis with spleen infarction and thrombosis in the superior and inferior mesenteric veins, with the size of the PNH clone at $82 \%$, when the diagnosis of PNH was established. Anticoagulation was initiated at diagnosis, first with low-molecular-weight heparin (LMWH), and then with warfarin. Eculizumab, in standard dose regimen, was initiated for the first time early on in 2015 together with warfarin. With the earlier-described therapy, haemolysis was well controlled-haemoglobin ranged from 107 to $155 \mathrm{~g} / \mathrm{L}$ with three compensated haemolytic crises during drug shortages with no subsequent thrombotic events (international normalized ratio [INR] was in the range of $2-3$ more than $80 \%$ of the time; D-dimer was constant at $<0.5 \mathrm{mg} / \mathrm{L}$ ).

Now 33 years old, our patient presented in the second quarter of 2020 with swelling and pain in left leg and lower back pain followed by episode of subfebrility $\left(37^{\circ} \mathrm{C}\right) 10$ days after the last dose of eculizumab. Co-amoxiclav and ciprofloxacin were started. Colour-duplex scan of lower limbs revealed proximal thrombosis of left popliteal vein. Nadroparin in full therapeutic dose was introduced (85.5 anti-Xa IU/kg SC BID). Laboratory tests showed decreased white blood cells $\left(3.4 \times 10^{9} / \mathrm{L}\right.$; with absolute neutrophil count $1.4 \times 10^{9} / \mathrm{L}$ and absolute lymphocyte count $1.4 \times 10^{9} / \mathrm{L}$ ), mild thrombocytopaenia $\left(140 \times 10^{9} / \mathrm{L}\right)$, mild haemolytic anaemia with haemoglobin $(113 \mathrm{~g} / \mathrm{L})$, absolute reticulocyte count $\left(265.5 \times 10^{9} / \mathrm{L}\right.$; normal: $\left.50-100 \times 10^{9} / \mathrm{L}\right)$, lactate dehydrogenase $(1,020 \mathrm{U} / \mathrm{L})$; normal $<460)$, and absent haptoglobin. C-reactive protein was elevated at $36.4 \mathrm{mg} / \mathrm{L}$ (normal $<10$ ), as well as D-dimer at $4.3 \mathrm{mg} / \mathrm{L}($ normal $<0.5)$; active partial thromboplastin time was prolonged ( 43.7 seconds; normal range: 25.1-36.5), while fibrinogen was in normal range at $5.12 \mathrm{~g} / \mathrm{L}$ (normal: $2.5-5.5$ ). His INR value at the time of thrombosis occurrence was 2 , while earlier values were in the range of 2 to 3 . The next dose of eculizumab was given 5 days after thrombosis occurrence according to regular schedule. Nasopharyngeal swab RNA PCR showed SARSCoV-2 positivity. The patient was afebrile and without respiratory symptoms throughout the period of thrombosis and follow-up. After 3 weeks, nadroparin was substituted with warfarin, with no recurrence of thrombosis in the subsequent 11 months.

\section{Discussion}

We present a patient with well-controlled PNH on eculizumab who developed DVT, while on warfarin, as the initial presentation of COVID-19. Meta-analysis of 1,988 hospitalized COVID-19 patients showed that prevalence of DVT was 19.8\% (95\% confidence interval [CI]: 10.5-34\%). However, while including only studies of patients on antithrombotic prophylaxis, DVT prevalence was $8 \%(95 \% \mathrm{CI}: 2.3-2.4 \%) .{ }^{5}$ While the majority of thrombotic cases were registered in hospitalized patients with COVID-19 pneumonia, up to this date only few cases of VTE as the presenting symptom of COVID-19 in non-PNH patients were reported. ${ }^{7-11}$ Putative risk factors for COVID-19 thrombosis are as follows: hyperinflammatory state, D-dimer greater than $1.5 \mathrm{mg} / \mathrm{L}$, hypoxia, need for invasive mechanical ventilation, immobilisation, advanced age, increased body mass index, and male sex. ${ }^{12-16}$ Besides male sex, in our patient, $\mathrm{PNH}$ as a prothrombotic state must be taken into account, with cumulative thrombotic incidence of 23 to $30 \%$ in pre-eculizumab era. ${ }^{17}$ However, initiation of eculizumab reduced thrombotic events in PNH by $81.8 \%$, with 0.62 events per 100 patient-years. ${ }^{17,18}$ Although rare, complementactivating events such as viral infection can provoke haemolysis and thrombotic events in PNH patients despite eculizumab therapy. ${ }^{19}$ Moreover, virus-induced complement activation and production of prothrombotic antibodies via a complement-dependent pathway ${ }^{20}$ could be a potential mechanism by which SARS-CoV-2 leads to immunothrombosis in patients susceptible to complement activation, as in PNH patients. Occasional reports of PNH patients with COVID-19 show that patients on anticomplement therapy did not develop pneumonia, while others (treatment naive) did, suggesting protective properties of anticomplement drugs in COVID19. ${ }^{19,21,22}$ Also, none of the reported PNH patients developed VTE. ${ }^{19,21,22}$ In light of this, several ongoing studies are evaluating eculizumab and other anticomplement therapies in severe COVID-19 patients with initial promising results. ${ }^{23-27}$ Consistent with previous results, our patient did not develop pneumonia. Given that our patient had long period with stable PNH disease, without thrombosis on eculizumab, and that anticoagulation was mainly in therapeutic range, we propose that SARS-CoV-2 infection outweighed the protective effects of anticomplement and anticoagulant therapy. To the best of our knowledge, this is the first case describing DVT in a PNH COVID-19 patient. This reveals an open debate on several issues. In well-anticoagulated patients, should the development of VTE, during the COVID-19 epidemic, raise a red flag for SARS-CoV-2 testing? Also, considering hypercoagulable effect of SARS-CoV-2, in patients with VTE risk factors, is there a need for more intensified anticoagulation? Given the variability of INR in patients on warfarin, is it preferable to use LMWH or even direct-acting anticoagulants which are not registered in this setting?

\section{Conflict of Interest}

The authors declare that they have no conflict of interest.

Acknowledgments

We would like to thank Slobodan Pilipovic, MD, for help in final language editing.

\section{References}

1 Risitano AM, Rotoli B. Paroxysmal nocturnal hemoglobinuria: pathophysiology, natural history and treatment options in the era of biological agents. Biologics 2008;2(02):205-222

2 Luzzatto L, Gianfaldoni G, Notaro R. Management of paroxysmal nocturnal haemoglobinuria: a personal view. Br J Haematol 2011; 153(06):709-720 
3 Pu JJ, Brodsky RA. Paroxysmal nocturnal hemoglobinuria from bench to bedside. Clin Transl Sci 2011;4(03):219-224

4 Lodigiani C, Iapichino G, Carenzo L, et al; Humanitas COVID-19 Task Force. Venous and arterial thromboembolic complications in COVID-19 patients admitted to an academic hospital in Milan, Italy. Thromb Res 2020;191:9-14

5 Di Minno A, Ambrosino P, Calcaterra I, Di Minno MND. COVID-19 and venous thromboembolism: a meta-analysis of literature studies. Semin Thromb Hemost 2020;46(07):763-771

6 Santoliquido A, Porfidia A, Nesci A, et al; Gemelli Against COVID19 Group. Incidence of deep vein thrombosis among non-ICU patients hospitalized for COVID-19 despite pharmacological thromboprophylaxis. J Thromb Haemost 2020;18(09): 2358-2363

7 Hughes C, Nichols T, Pike M, Subbe C, Elghenzai S. Cerebral venous sinus thrombosis as a presentation of COVID-19. Eur J Case Rep Intern Med 2020;7(05):001691

8 Hemasian H, Ansari B. First case of COVID-19 presented with cerebral venous thrombosis: a rare and dreaded case. Rev Neurol (Paris) 2020;176(06):521-523

9 Davoodi L, Jafarpour H, Taghavi M, Razavi A. COVID-19 presented with deep vein thrombosis: an unusual presenting. J Investig Med High Impact Case Rep 2020;8:2324709620931239

10 Mohammadi S, Abouzaripour M, Hesam Shariati N, Hesam Shariati MB. Ovarian vein thrombosis after coronavirus disease (COVID-19) infection in a pregnant woman: case report. J Thromb Thrombolysis 2020;50(03):604-607

11 Veyseh M, Pophali P, Jayarangaiah A, Kumar A. Left gonadal vein thrombosis in a patient with COVID-19-associated coagulopathy. BMJ Case Rep 2020;13(09):e236786

12 Rali P, O'Corragain O, Oresanya L, et al. Incidence of venous thromboembolism in coronavirus disease 2019: an experience from a single large academic center. J Vasc Surg Venous Lymphat Disord 2021;9(03):585-591.e2

13 Cui S, Chen S, Li X, Liu S, Wang F. Prevalence of venous thromboembolism in patients with severe novel coronavirus pneumonia.J Thromb Haemost 2020;18(06):1421-1424

14 Miesbach W, Makris M. COVID-19: coagulopathy, risk of thrombosis, and the rationale for anticoagulation. Clin Appl Thromb Hemost 2020;26:1076029620938149

15 Fontana P, Casini A, Robert-Ebadi H, Glauser F, Righini M, Blondon M. Venous thromboembolism in COVID-19: systematic review of reported risks and current guidelines. Swiss Med Wkly 2020;150: w20301
16 Dobesh PP, Trujillo TC. Coagulopathy, venous thromboembolism, and anticoagulation in patients with COVID-19. Pharmacotherapy 2020;40(11):1130-1151

17 Griffin M, Munir T. Management of thrombosis in paroxysmal nocturnal hemoglobinuria: a clinician's guide. Ther Adv Hematol 2017;8(03):119-126

18 Malato A, Saccullo G, Coco LL, et al. Thrombotic complications in paroxysmal nocturnal haemoglobinuria: a literature review. Blood Transfus 2012;10(04):428-435

19 Kulasekararaj AG, Lazana I, Large J, et al. Terminal complement inhibition dampens the inflammation during COVID-19. Br J Haematol 2020;190(03):e141-e143

20 Valerio L, Riva N. Head, neck, and abdominopelvic septic thrombophlebitis: current evidence and challenges in diagnosis and treatment. Hamostaseologie 2020;40(03):301-310

21 Shikdar S, Borogovac A, Mohamad E, Khawandanah M. COVID-19 infection in a patient undergoing treatment for paroxysmal nocturnal hemoglobinuria (PNH) with ravulizumab [preprint, version 1]. 2020. Accessed November 16, 2020 at: https://doi. org/10.21203/rs.3.rs-84187/v1

22 Schüller H, Klein F, Lübbert M, et al. Hemolytic crisis in a patient treated with eculizumab for paroxysmal nocturnal hemoglobinuria possibly triggered by SARS-CoV-2 (COVID-19): a case report. Ann Hematol 2020;100(03):841-842

23 Diurno F, Numis FG, Porta G, et al. Eculizumab treatment in patients with COVID-19: preliminary results from real life ASL Napoli 2 Nord experience. Eur Rev Med Pharmacol Sci 2020;24(07):4040-4047

24 Vlaar APJ, de Bruin S, Busch M, et al. Anti-C5a antibody IFX-1 (vilobelimab) treatment versus best supportive care for patients with severe COVID-19 (PANAMO): an exploratory, open-label, phase 2 randomised controlled trial. Lancet Rheumatol 2020;2 (12): e764-e773

25 Clinicaltrials.Gov. CORIMUNO19-ECU: Trial Evaluating Efficacy and Safety of Eculizumab (Soliris) in Patients with COVID-19 Infection, Nested in the CORIMUNO-19 Cohort (CORIMUNO19ECU). 2020. Accessed November 22, 2020 at: https:// clinicaltrials.gov/ct2/show/NCT04346797

26 Mastellos DC, Pires da Silva BGP, Fonseca BAL, et al. Complement C3 vs C5 inhibition in severe COVID-19: early clinical findings reveal differential biological efficacy. Clin Immunol 2020;220:108598

27 Annane D, Heming N, Grimaldi-Bensouda L, et al; Garches COVID 19 Collaborative Group. Eculizumab as an emergency treatment for adult patients with severe COVID-19 in the intensive care unit: a proof-of-concept study. EClinicalMedicine 2020;28:100590 